\title{
Posteromedial Plate as Strategy for Fixation of Posterior Column of Tibial Plateau Fracture
}

Adnan El Sebaie ${ }^{1}$ MD, Ahmed El Geushy ${ }^{1}$ MD, Mohamed Sabry ${ }^{1 *}$ M.B.B.C.H

*CorrespondingAuthor:Mohamed

Sabry

mohamedsabry5001@gmail.com

Received for publication

February18, 2020;

AcceptedMay10, 2020; Published

on line June03, 2020.

Copyright 2020 The Authors published by Al-Azhar University, Faculty of Medicine, Cairo, Egypt. All rights reserved. This an openaccess article distributed under the legal terms, where it is permissible to download and share the work provided it is properly cited. The work cannot be changed in anyway or used commercially.

doi:10.21608/aimj.2020.23545.113

3

${ }^{1}$ Orthopedic Surgery Department, Faculty of Medicine, Al-Azhar University, Cairo, Egypt

Disclosure: The authors have no financial interest to declare in relation to the content of this article. The Article Processing Charge was paid for by the authors.

\section{Authorship: All authors have}

a substantial contributions to the article

\begin{abstract}
Background:A posteromedial tibial plateau fracture is considered a challenging injury pattern of the tibial plateau. Fracture line lies in the coronal plane leading to separation of a posteromedial fragment of variable size. This type of fracture is not rare, but it has been underappreciated previously.This study is aiming to spot light on posteromedial approach as new concept in treatment of tibial plateau fracture as regarding to clinical and radiological outcome and to assess its surgical efficacy and advantages in such cases and to show is it beneficial to the patients or not?
\end{abstract}

Patient and Methods:This is a prospective study that included 15 consecutive patients (13 males \& 2 females) with closed posteromedial tibial plateau fractures who had undergone open reduction and internal fixation through a posteromedial approach by plate and screws between May 2019 and September 2019. Our protocol in follow up ranged from three to six months postoperative.

Results:In our study, the total clinical score according to the Rasmussen system was excellent in 8 patients (53.3\%) and good in 7 patients (46.7\%). As for complications, one case presented at the 3rd day postoperative with signs of compartment syndrome followed by superficial infection. The patient improved with conservative treatment with no need for surgical intervention.

Conclusion:The posteromedial approach in managing posteromedial tibial plateau fracture is considered a safe and efficient approach. It allows perfect visualization and reduction of the displaced fragments. This approach uses an intermuscular plane without exposure of the neurovascular bundle.

Key words:Posteromedial Plate; Tibial Plateau Fracture; Fixation of PosteriorColumn

failure to expose the fracture site, impairment of the blood supply of the soft tissues, and risk of injuring the neurovascular bundles. ${ }^{4}$

The posteromedial approach was first described in a German literature in 2003 and is usually used to access the posteromedial and posterior side of the tibial plateau. It allows for good visualization and reduction of the posteromedial fragment without exposure of the neurovascular bundle. ${ }^{5}$

Our study is aiming to spot light on the posteromedial approach in managing the posteromedial tibial plateau fractures, and to evaluate the patients clinically and radiologically after fixation of the fracture with a buttress plate.

\section{PATIENTS AND METHODS}

This is a prospective study that included 15patients (13males \& 2 females) with closed posteromedial tibial plateau fractures who had undergone surgery in the period between May 2019 and September 2019. The protocol was discussed and approved for clinical study by the Ethical Research Committee of Al-Azhar University and a written informed consent was obtained. All patients were informed about the pathology and the suggested treatment according to their diagnosis and also informed about the possible tibial plateau is one of the most challenging types of the tibial plateau fractures. ${ }^{1}$ This type of fracture is previously. Attention to these types of fractures markedly increased with the advances achieved in computed tomography (CT) technology. ${ }^{2}$ These fractures are usually caused by high-energy trauma commonly results in articular surface soft-tissue injury. ${ }^{3}$

Several surgical approaches like the anterolateral approach, the anteromedial approach and the posterior approach have been prescribed. However, each approach has its shortcoming including damage of the soft tissues caused by dissection, 
complications. All our patients underwent the same treatment of open reduction and internal fixation by a buttress plate and screws to fix the posteromedial tibial plateau fracture through a posteromedial approach at Bab AL-Sharia (SayedGalal) university hospital and Dar El Shefa Hospital, Cairo, Egypt. The average patient age at the operation time was 34.8 years (age ranges from 20 to 48 years). Our follow up period extended from three to six months. According to the Schatzker classification, there were 6 cases (40\%) with type lVfractureand9 cases (60\%) with type Vfracture.

Inclusion Criteria were: Posteromedial Column Fracture, Schatzker Type IV and V BicondylarTibial Plateau Fracture, Moore Type 1 posterior Split Fracture. We excluded patients with Open fractures, Neurovascular Injury, Schatzker type VI

Indication for surgery in tibial plateau fracture was described by Honkonen suggesting that surgical treatment should be considered in all fractures of the medial condyle, medially tilted bicondylar fracture, all fractures of the lateral condyle and laterally tilted bicondylar fractures showing more than 5 degrees of lateral tilt, more than $3 \mathrm{~mm}$ of articular step-off, or condylar widening greater than $5 \mathrm{~mm}$, in addition to all displaced axial bicondylar fractures.

\section{Surgical procedure:}

Patients were operated under general or spinal anesthesia. All Patients were placed in the prone position with the hip laterally tilted on a radiolucent operative table, with the other leg extended on a leg holder or any other similar positioning device. The administration of preoperative antibiotic was done after sensitivity test, the leg is then exsanguinated and a tourniquet was inflated around the upper thigh.

Surgical landmarks in the posteromedial approach consist of the medial head of the hamstrings and gastrocnemius muscle, which are palpated on the medial and distal border of the popliteal fossa. We made an incision about $10 \mathrm{~cm}$ along the border of the medial head of the gastrocnemius muscle.

Sharp dissection is done in the subcutaneous tissue and popliteal fascia using a blade knife (Figure 1A). After that we identified and bluntly dissected the medial head of the gastrocnemius muscle from distal to proximal and retracted it laterally. The insertion of the hamstrings (pes anserine tendons) is then retracted medially revealing the popliteus muscle underneath which is dissected completely or partially and detached from the proximal tibia subperiosteally with a periosteal elevator.

Hohmann retractor is then placed subperiosteally and the periosteum is then incised carefully and elevated with caution to reveal the fracture site. Indirect reduction can be achieved through hyperextension with axial traction, compression on the fragment and applying a T-shaped buttress plate, with the first screw placed through the plate and into an oblong hole just distal to the fracture. this allowed the reduction of the fracture by approximating the plate to the bone (Figure 1B).

Wires can be used to temporary fix the plate in place. Using image intensifier in the lateral view ensured perfect proximal level of the plate. After that the rest of the screws are inserted through the plate (Figure 2).

The tourniquet was turned off slowly before wound closure, vascularity was checked and small vessels were cauterized. Closure of the wound was done by closing the deep fascia, subcutaneous tissue and skin. A $12 \mathrm{~mm}$ drain was inserted. The wound was then covered with a sterile dressing.

In patients with bicondylar fractures that needs fixation of the lateral tibial condyle, the leg is prepared and draped again for a standard approach to fracture fixation either by an anterolateral approach to apply an anterolateral plate or with percutaneous $6.5 \mathrm{~mm}$ or $7.3 \mathrm{~mm}$ cannulated lag screws (Figure 3).

\section{Post-operative stage:}

Proper analgesia was continued for the first 2 days, anti-edematous measures were done in the form of elevation of the operated limb and cold packs for 24 hours. Physiotherapy started on the first postoperative day in the form of chest exercise and cold therapy. In patients with unicondylar fractures, continuous passive motion was started on the first post-operative day. In cases with bicondylar fractures, posterior above knee splint was done and range of motion was delayed for 10 to 14 days. On the third post-operative day, all patients were allowed to walk using two crutches with no weight bearing to the affected limb. Hospital stay ranged from 2 to 5 days postoperatively

Follow up:

Two weeks follow up was done to evaluate the wound, remove stitches and check for complication like deep venous thrombosis and infection. Eight weeks follow up was done to check the fracture union and plan for the weight bearing. Twelve weeks follow up was done to assess the clinical and radiological outcome according to the Rasmussen system (Table 1).

Clinical score is considered Excellent with 18 points, good from 12 to 18 points, fair from 6 to 11 points and poor with less than 6 points.Radiological score is evaluated asexcellent with 18 points, good from 12 to 18 points,fair from 6 to 11 pointsandpoor with less than 6 points.

\section{Statistical Analysis:}

Data were collected, revised, coded and entered to the Statistical Package for Social Science (IBM SPSS) version 23. The quantitative data were presented as mean, standard deviations and ranges when their distribution found parametric and median, inter-quartile range (IQR) when data found non parametric. Also, qualitative variables were presented as number and percentages. So, the p- 
value was considered significant as the following: $\mathrm{P}$ $>0.05$ : Non significant, $\mathrm{P}<0.05$ : Significant, $\mathrm{P}<$ 0.01: Highly significant.

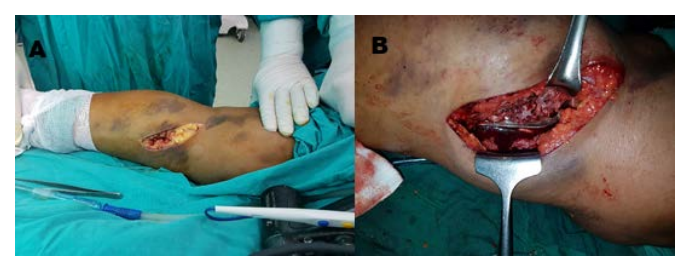

Fig (1):posteromedial approach to the tibial plateau. (A) sharp dissection is done in the subcutanous tissue and poplteal fascia using a blade knife medial to the medial head of the gastrocnemius. (B) conventional nonlocking $(4.5-\mathrm{mm})$ buttress $\mathrm{T}$ plate used to fix the posteromedial fragment.

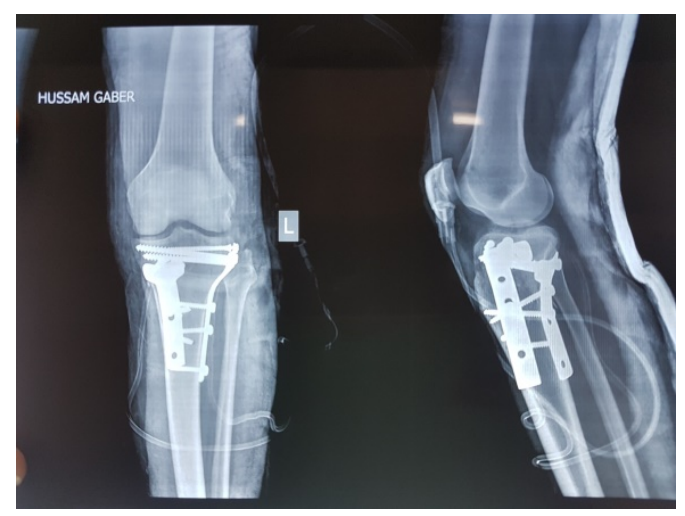

Fig(2): bicondylartibial plateau fracture fixed by double plating.

\section{RESULTS}

Our study included 15 patients, 2 of them were females and 13 were males with age ranged from 20 years to 48 years and a mean age at the time of the injury of 34.8 years. A total number of 8 patients (53.3\%) presented by tibial plateau fracture in the right limb and 7 patients (46.7\%) in the left limb.

There was significant correlation between the type of fracture and the total radiological score. A total of 6 patients with type IV fracture had a mean radiological score of 18 points while 9 patients with type $\mathrm{V}$ fracture had a mean radiological score of 14 points. Better radiological results were observed in patients with type IV fracture (Table 2).

In this study, we included 6 patients with fracture type IV, 4 of them (66.6\%) had excellent score and 2 of them (33.4\%) had good score. The other 9 patients had fracture type V, 4 of them (44.4\%) had excellent score while 5 of them (55.6\%) had good results (Table 3).

In this study, one case presented at the 3rd day postoperative with signs of compartment syndrome. The patient was managed by close observation and conservative treatment that included massive antiedematous, leg elevation and cold packs. On the 6th day, the same patient showed dehiscence and superficial infection on the distal 3 sutures. Culture and sensitivity tests were done and the proper antibiotic was administrated for two weeks. The patient improved with conservative treatment with no need for surgical intervention.

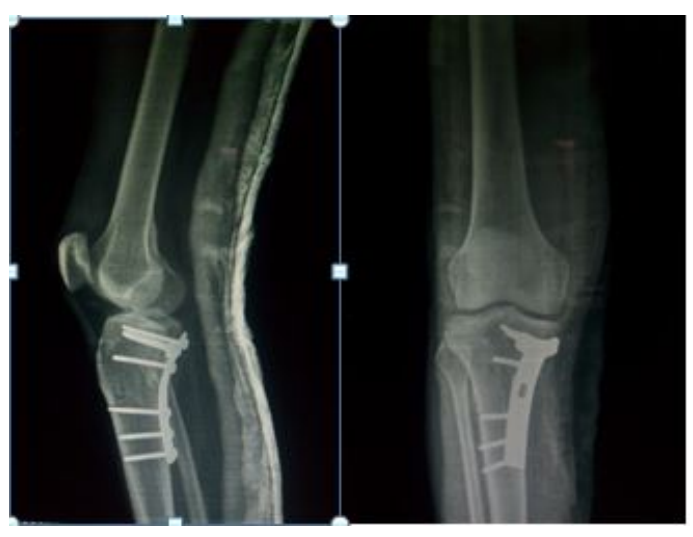

Fig (3): posteromedial unicondylartibial plateau fracture fixed by posteromedial plate. 


\begin{tabular}{|c|c|c|c|}
\hline Clinical Parameter & Points & Radiological Parameter & Points \\
\hline Subjective & & Depression & \\
\hline Pain & & None & 6 \\
\hline None & 6 & $<6 \mathrm{~mm}$ & 4 \\
\hline Occasional pain, needs no medication & 5 & $6-10 \mathrm{~mm}$ & 2 \\
\hline Stabbing pain & 4 & $>10 \mathrm{~mm}$ & 0 \\
\hline Intense, activity-related & 2 & Condylar widening & \\
\hline Night pain, at rest & 0 & None & 6 \\
\hline Walking capacity & & $<6 \mathrm{~mm}$ & 4 \\
\hline Normal & 6 & $6-10 \mathrm{~mm}$ & 2 \\
\hline Outdoors $>1 \mathrm{~h}$ & 4 & $>10 \mathrm{~mm}$ & 0 \\
\hline Outdoors $>15 \mathrm{~min}$ & 2 & Angulation (valgus/varus) & \\
\hline Indoors only & 1 & None & 6 \\
\hline Wheelchair/bedridden & 0 & $<10^{\circ}$ & 4 \\
\hline Objective & & $10^{\circ}-20^{\circ}$ & 2 \\
\hline Extension & & $>20^{\circ}$ & 0 \\
\hline Normal & 6 & Total (minimum) & \\
\hline$<10^{\circ}$ loss & 4 & & \\
\hline$>10^{\circ}$ loss & 2 & & \\
\hline \multicolumn{2}{|l|}{ Total range of motion } & & \\
\hline$>140^{\circ}$ & 6 & & \\
\hline$>120^{\circ}$ & 5 & & \\
\hline$>90^{\circ}$ & 4 & & \\
\hline$>60^{\circ}$ & 2 & & \\
\hline$>30^{\circ}$ & 1 & & \\
\hline $0^{\circ}$ & 0 & & \\
\hline \multicolumn{2}{|l|}{ Stability } & & \\
\hline Normal & 6 & & \\
\hline Abnormal in $20^{\circ}$ flexion & 5 & & \\
\hline Instability in extension $<10^{\circ}$ & 4 & & \\
\hline Instability in extension $>10^{\circ}$ & 2 & & \\
\hline Total & & & \\
\hline
\end{tabular}

Table 1: Rasmussen Score (Criteria of Clinical and radiological assessment)

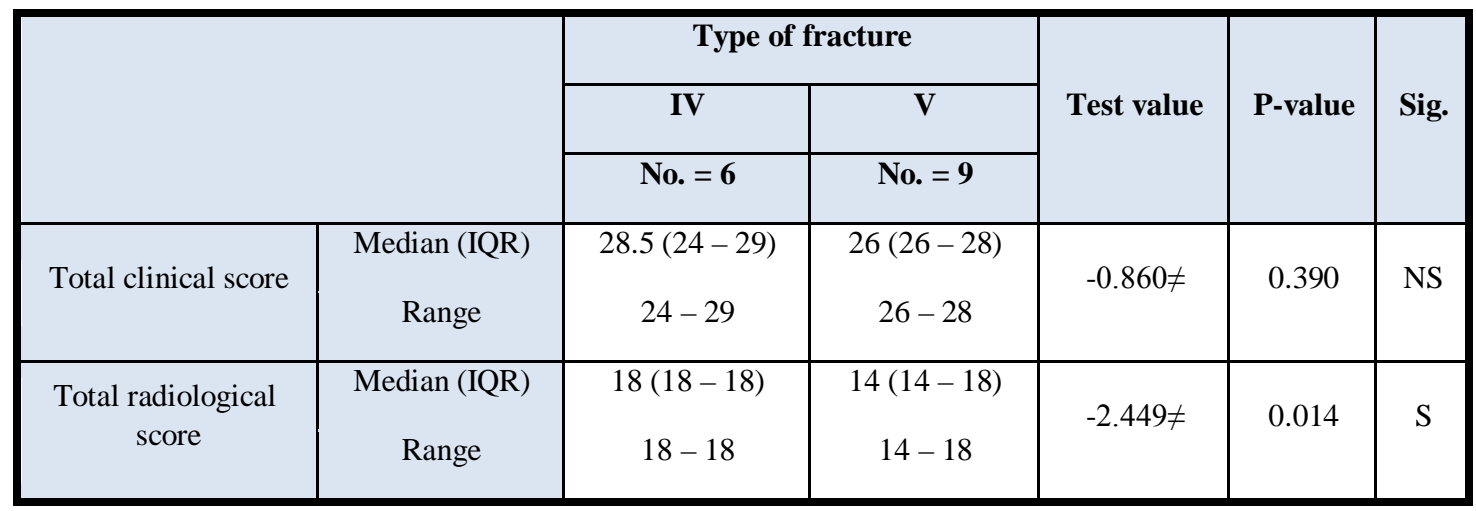

Table 2: Clinical and radiological outcome in relation to the Type of fracture

P-value > 0.05: Non significant; P-value < 0.05: Significant; P-value < 0.01: Highly significant 


\begin{tabular}{|c|c|c|c|c|c|}
\hline & & & \multicolumn{2}{|c|}{ Clinical Result } & \multirow[t]{2}{*}{ Total } \\
\hline & & & Excellent & Good & \\
\hline \multirow{4}{*}{ Type of Fracture } & \multirow{2}{*}{ IV } & Count & 4 & 2 & 6 \\
\hline & & \% within Type of Fracture & $66.6 \%$ & $33.4 \%$ & $100.00 \%$ \\
\hline & \multirow{2}{*}{$\mathbf{V}$} & Count & 4 & 5 & 9 \\
\hline & & \% within Type of Fracture & $44.4 \%$ & 55.6 & $100.00 \%$ \\
\hline & & Total count & 8 & 7 & $100.00 \%$ \\
\hline & & \% within Type of Fracture & $53.3 \%$ & $46.7 \%$ & \\
\hline
\end{tabular}

Table 3: Percentage of the clinical outcome according to each fracture type.

\section{DISCUSSION}

The incidence of Posteromedial split fracture of the proximal tibial is relatively uncommon. The AO and Schatzker classifications failed to describe it because they do not differentiate when the fracture of the medial condyle is mainly posterior and may be associated with a dislocation as the Moore system does.

The anteromedial approach does not allow direct visualization of the fracture, so it will be very difficult to manipulate fragment. In order to visualize the fragment, extensive medial dissection is required.Direct posterior approach is more difficult and riskier as it requires complete dissection of the neurovascular bundle in the popliteal fossa.

In the study conducted by Hsieh et al. ${ }^{4}$, eight patients with fractures of the posteromedial tibia plateau were included. All patients were treated with fracture reduction using an anteromedial approach and fixation by cancellous screws from anterior to posterior. Although the results were satisfactory but the reduction was difficult, consumed more time and radiation exposure. The fixation was not ideal and the fracture union was delayed.

In the study conducted by Lee et al. ${ }^{6}$, he included fifteen patients with bicondylar plateau fracture treated by open reduction and fixation with unilateral locked plate. Five patients (33.3\%) were classified as excellent while the remaining 10 (66.7\%) were classified as good. Mal-reduction occurred in one patient (6.7\%) because of inadequate reduction of the posteromedial fragment. Fixation loss was observed in three patients when partial weightbearing commenced. Fixation loss was mainly due to subsidence of the posteromedial fragment.

The posteromedial approach in prone position is considered the best option for treating types of fractures. In our study we used this approach because it does not include dissection of the neurovascular bundle. It also allows direct visualization of the fracture pattern, appropriate placement of posterior antiglide buttress plate and does not require extensive soft tissue dissection. Furthermore, the prone positioning greatly facilitates the reduction by applying axial traction with hyperextension of the knee.

In our study, there was significant correlation between the type of fracture and the total radiological score. A total of 6 patients with type IV fracture had a mean radiological score of 18 while 9 patients with type $\mathrm{V}$ fracture had a mean radiological score of 14 points. In a study conducted by Chiu et al ${ }^{7}$, the correlation of the results to the type of the fracture (types IV, V, and VI) were not statistically significant.

In our study, six out of fifteen patients presented by schatzker type IV fracture. The clinical Rasmussen score of this group was $66.6 \%$ excellent, $33.4 \%$ was good, the other nine patients included with schatzker type $\mathrm{V}$ fracture, the score of this group was $44.4 \%$ excellent and $55.6 \%$ was good. The total clinical score according to the Rasmussen system was excellent in 8 patients (53.3\%) and good in 7 patients $(46.7 \%)$. All patients were satisfied by the net results and could return to their daily activity.

In the study conducted by Chen et al. ${ }^{8}$, Thirty-six patients with posteromedial tibial plateau fracture managed by the described method of fixation.. The results were graded as excellent in 21 cases, good in 13 cases, fair in 2 cases. The rate of excellent and good results was (94.4\%).

In a study conducted by Chiu et al. ${ }^{7}$ on Twenty-five patients with fracture of the posteromedial column of the tibial plateau managed by the described method of fixation. A total of 11 patients (44\%) had excellent scores, 12 of them (48\%) had good scores, and 2 patients (8\%) had fair results. In total, 23 patients 
representing $92 \%$ of the patients had satisfactory results.

In this study, one case presented at the 3rd day postoperative with signs of compartment syndrome followed by minor superficial wound infection. The patient was managed by close observation and conservative treatment. The patient improved with conservative treatment with no need for surgical intervention

Stahel and Mauffrey ${ }^{9}$, in their study with more than 50 patients, postoperative complications included one patient with a postoperative wound infection that required an unplanned surgical debridement and washout of the surgical wound. Three patients had excessively long posterior apex screws that required early plate removal after fracture union. One case with osteoporotic bone had a displacement of the fracture after 3 weeks from the injury.

In Chiu et al. $^{7}$ study, post-operative complications included One patient (4\%) with a Schatzker type VI fracture had a minor surgical wound dehiscence. The wound was managed successfully by continuous wound care and strong antibiotic therapy. Also, one patient with a fracture type VI had moderate osteoarthritis with pain and stiffness (range of motion, 10 to 90 degree). Depression of the joint surface of the tibial plateau was recorded in 3 patients (12\%) with no functional instability. Paresthesia on the lateral calf of the distal part of the leg was recorded in 5 patients (20\%).

\section{CONCLUSION}

The posteromedial approach is considered a safe and efficient approach. IT provides perfect visualization and reduction of the displaced fragments. It allows fixation of the posteromedial fragment with a posterior antiglide buttressing plate respecting the fracture biomechanics without exposure of the neurovascular bundle.

\section{CONFLICT OF INTEREST STATEMENT}

We certify that we have no affiliations with or involvement in any organization or entity with any financial interest or non-financial interest in the subject matter or materials discussed in this manuscript.

\section{ACKNOWLEDGMENTS}

I wish to express my deepest thanks and gratitude to prof. Adnan El Sebaie and Dr Ahmed El Geushy for their huge support during the study and to everyone who participated in this work.

\section{REFERENCES}

1. Babis GC, Evangelopoulos DS, Kontovazenitis $P$, et al. High energy tibial plateau fractures treated with hybrid external fixation. $J$ OrthopSurg Res.2011;6(1):1-7. doi:10.1186/1749-799X-6-35

2. Zhu Y, Yang G, Luo CF, et al. Computed tomography-based Three-Column Classification in tibial plateau fractures: Introduction of its utility and assessment of its reproducibility. $J$ Trauma Acute Care Surg. 2012;73(3):731-37. doi:10.1097/TA.0b013e31825c17e7

3. Conserva V, Vicenti G, Allegretti G, et al. Retrospective review of tibial plateau fractures treated by two methods without staging. Injury. 2015;46(10):1951-56.

doi:10.1016/j.injury.2015.07.018

4. Hsieh C-H, Huang H-T, Liu P-C,et al. Anterior approach for posteromedial tibial plateau fractures. Kaohsiung $J$ Med Sci. 2010;26(3):130-35.

doi:10.1016/S1607-551X(10)70019-X

5. Hake ME, Goulet JA. Open Reduction and Internal Fixation of the Tibial Plateau Through the Anterolateral Approach. J Orthop Trauma. 2016;30(8):S28-S29. doi:10.1097/BOT.0000000000000581

6. Lee $\mathrm{T}$, Huang $\mathrm{H}$, Lin $\mathrm{Y}$, et al. Bicondylartibial plateau fracture treated by open reduction and fixation with unilateral locked plating. Kaohsiung J Med Sci. 2013;29(10):568-77. doi:10.1016/j.kjms.2013.01.006

7. Chiu C-H, Cheng C-Y, Tsai M-C, et al. Arthroscopy-assisted reduction of posteromedial tibial plateau fractures with buttress plate and cannulated screw construct. Arthrosc $J$ ArthroscRelat Surg.2013;29(8):1346-54. doi:10.1016/j.arthro.2013.05.003

8. Chen HW, Liu GD, Ou S, et al. Open reduction and internal fixation of posterolateraltibial plateau fractures through fibula osteotomy-free posterolateral approach. $J$ Orthop Trauma. 2014;28(9):513-17. doi:10.1097/BOT.0000000000000047

9. Stahel, Philip F CM. Direct posterior approach for the treatment of posteromedial tibial head fractures. OrthopKnowl Online J. 2014;12:10. doi:10.1007/s00064-009-1605-y 